\title{
Rapamycin induces autophagy in the melanoma cell line M14 via regulation of the expression levels of Bcl-2 and Bax
}

\author{
XUE LI* ${ }^{*}$ DI WU* ${ }^{*}$ JINGGANG SHEN, MEIHUA ZHOU and YAN LU \\ Department of Dermatology, The First Affiliated Hospital of Nanjing Medical University, \\ Nanjing, Jiangsu 210029, P.R. China
}

Received March 14, 2012; Accepted October 9, 2012

DOI: $10.3892 / \mathrm{ol} .2012 .986$

\begin{abstract}
Cancer therapy with rapamycin has been successfully implemented for kidney cancer, glioblastoma and prostate cancer. However, there are few studies concerning the effects of rapamycin on the treatment of human melanoma. In this study, we investigated whether rapamycin may be a promising strategy for the effective treatment of melanoma and explored the possible mechanism for this by culturing M14 cells in vitro and treating with rapamycin at three concentrations $(10,50$ or $100 \mathrm{nmol} / \mathrm{l})$. MDC and LC3B staining, western blot analysis, flow cytometry and transmission electron microscopy were employed. We revealed that rapamycin induced autophagy and inhibited the proliferation of M14 cells in a concentration-dependent manner, Furthermore, western blot analysis revealed an upregulated expression of $\mathrm{Bcl}-2$ and downregulated expression of Bax in M14 cells. In conclusion, rapamycin induced autophagy and inhibited the growth of M14 cells. The mechanism may involve regulation of the expression of $\mathrm{Bcl}-2$ family proteins. Rapamycin appears to be a promising strategy for the effective treatment of melanoma.
\end{abstract}

\section{Introduction}

Autophagy is an active degradative process that removes or recycles bulk cytoplasmic constituents through the endosomal and lysosomal fusion system, resulting in the formation of autophagosomes in eukaryotic cells. The autophagic process is robustly upregulated in response to cellular stress, such as nutrient or cytokine depletion, hypoxia and oxidative damage, and it is also pivotal to innate intracellular defense mechanisms against certain pathogens. Autophagy has significant roles in tissue development, differentiation and remodeling (1).

Correspondence to: Dr Yan Lu, Department of Dermatology, the First Affiliated Hospital of Nanjing Medical University, Nanjing, Jiangsu 210029, P.R. China

E-mail: luyan1971@yahoo.com.cn

${ }^{*}$ Contributed equally

Key words: melanoma, rapamycin, autophagy, Bcl-2, Bax
It is also implicated in diseases such as in the development of tumors, although its precise role is ambiguous (2).

Melanoma is the most fatal form of skin cancer with increasing incidence throughout the world. There are no efficacious therapies for malignant melanoma at present (3). The alkylating agent dacarbazine, administered as a single agent, remains the current standard treatment. However, few patients are capable of achieving remission from distant metastases and the 5-year survival rate is $10 \%$. Thus, new agents and/or therapeutic strategies with different action targets need to be developed.

Rapamycin, a lipophilic macrolide antibiotic, was originally identified as a fungicide and immunosuppressant (4). However, studies have revealed that rapamycin can potently arrest the growth of cells derived from a broad spectrum of cancers (5). Rapamycin has been shown to specifically inhibit its target, mammalian target of rapamycin (mTOR), which plays a key role in tumor development and progression. Rapamycin binds the immunophilin FK506 binding protein (FKBP12) to form the FKBP12-rapamycin complex, which then interacts with mTOR and inhibits the mTOR-mediated phosphorylation of S6K1 and 4E-BP1. In addition, rapamycin is the best characterized drug that enhances autophagy, a process of 'self-eating' that involves both the death and survival of cancer cells. Therefore, rapamycin may interfere with different aspects of the tumor. Certain authors have demonstrated that rapamycin inhibits lung metastasis of B16 melanoma cells through downregulating alphav integrin expression and upregulating apoptosis signaling; autophagy is not involved in the rapamycin-mediated suppression of metastasis (6). However, there are few studies concerning the effects of rapamycin on human melanoma and the interaction with autophagy, thus the impact of rapamycin on M14 cells remains unclear.

Bcl-2 family proteins, which have either pro- or anti-apoptotic activities, have been studied intensively for the past decade owing to their significance in the regulation of apoptosis, tumorigenesis and cellular responses to anti-cancer therapy (7). Aberrant expression of Bcl-2 family members is capable of inappropriately promoting or preventing apoptosis. Bcl-2 is an anti-apoptotic member that prevents the release of cytochrome $\mathrm{c}$ from the mitochondrial intermembrane space (IMS) into the cytosol. Oppositely, Bax is a cytosolic protein that translocates to the mitochondria and participates in the release of cytochrome $\mathrm{c}$ in response to apoptotic stimuli. There 
is a negative correlation between the expression of Bcl-2 and Bax. In short, Bcl-2 overexpression leads to cell survival and Bax overexpression results in cell death (8).

Morever, Bcl-2 family proteins also target the autophagy pathway. In this study, we set out to observe the autophagy of M14 cells induced by rapamycin; to investigate the effects of rapamycin on regulating the expression of Bcl-2 and Bax and to identify whether rapamycin may be a promising strategy for the effective treatment of melanoma.

\section{Materials and methods}

Cell culture. The human melanoma cell line M14 was obtained from Fuxiang Bio-Technology Company (Shanghai, China). Cells were maintained at $37^{\circ} \mathrm{C}$ and $5 \% \mathrm{CO}_{2}$ in Dulbecco's modified Eagle's medium (DMEM; Gibco, Carlsbad, CA, USA) supplemented with $10 \%(\mathrm{v} / \mathrm{v})$ fetal bovine serum (FBS) and $1 \%$ penicillin/streptomycin (Gibco). Cells were inoculated at a density of $1 \times 10^{5}$ cells $/ \mathrm{ml}$ and grown for 3 days to reach a phase of exponential growth (log phase), and were then used for the majority of experiments unless specified otherwise.

Autophagy induction and reagent treatment. Cells were plated at a density of approximately $1 \times 10^{5}$ viable cells/well in 96-well microtiter plates. For autophagy induction, cells were treated with or without rapamycin $(10,50$ or $100 \mathrm{nmol} / \mathrm{l})$ for $24 \mathrm{~h}$. Following treatment, cells were analyzed as subsequently described.

Monodansylcadaverine (MDC) labeling. Cells were grown on chamber slides, washed with phosphate-buffered saline (PBS) and fixed in $10 \%$ formalin solution for $10 \mathrm{~min}$. Autophagic vacuoles were labeled with MDC by incubating cells with $0.05 \mathrm{mmol} / \mathrm{l} \mathrm{MDC}$ (Sigma-Aldrich, St. Louis, MO, USA) in PBS at $37^{\circ} \mathrm{C}$ for $10 \mathrm{~min}$. Following incubation, cells were washed three times with PBS and immediately analyzed under a fluorescence microscope (BX50, Olympus).

Immunofluorescent staining of LC3B. M14 cells ( $1 \times 10^{5} /$ well) were seeded on glass cover slides in 24-well plates and left to attach overnight. Cells were then treated for $24 \mathrm{~h}$ with 10 , 50 or $100 \mathrm{nmol} / 1$ rapamycin. Cells were then washed with PBS and fixed with 4\% paraformaldehyde in PBS for 15 min at room temperature. Subsequently, they were permeabilized by $100 \mu \mathrm{g} / \mathrm{ml}$ digitonin for $15 \mathrm{~min}$, followed by PBS with $3 \%$ bovine serum albumin (BSA) for $1 \mathrm{~h}$ at room temperature. The anti-LC3B antibody was diluted to 1:200 in PBS, which contained $1 \%$ BSA, and then co-incubated with cells overnight at $4^{\circ} \mathrm{C}$. After washing twice with PBS, cells were then incubated with Cy3-labeled goat anti-rabbit IgG (Sigma, St. Louis, MO, USA) for $1 \mathrm{~h}$ at room temperature in the dark. Then, cells were washed three times with PBS and stained with 4',6-diamidino-2-phenylindole (DAPI; $10 \mu \mathrm{g} / \mathrm{ml}$ ) for $5 \mathrm{~min}$. Finally, samples were imaged under a confocal fluorescence microscope (BX50, Olympus).

\section{3-(4,5-dimethylthiazol-2-yl)-2,5-diphenyltetrazolium}

bromide (MTT) analyses. Cell viability was measured by MTT assay (Amresco, Solon, Ohio, USA). M14 cells $\left(1 \times 10^{5} / 100 \mu \mathrm{l}\right)$ were seeded in $96-w e l l$ plates. Following treat- ment, $20 \mu 1$ of MTT solution $(5 \mathrm{mg} / \mathrm{ml})$ was added to each well and incubated (at $37^{\circ} \mathrm{C}$ and $5 \% \mathrm{CO}_{2}$ ) for $4 \mathrm{~h}$. Next, the medium was removed and the wells were allowed to dry. The MTT metabolic product was resuspended in $200 \mu \mathrm{l}$ of dimethylsulfoxide (DMSO) and placed on a shaking table for $5 \mathrm{~min}$. At this point, the absorbance (optical density) was measured at $530 \mathrm{~nm}$ using a microplate reader. The cell proliferation inhibition rate was calculated using the equation: Inhibition rate of proliferation $(\%)=\left(\mathrm{A}_{\text {control }}-\mathrm{A}_{\text {experimental }}\right) /$ $\mathrm{A}_{\text {control }} \times 100$ (1).

Western blot analyses for Bcl-2 and Bax. Cellular lysate was prepared using radioimmunoprecipitation assay (RIPA) buffer with protease inhibitors and quantified using the bicinchoninic acid (BCA) protein assay (Pierce Biotechnology Inc., Rockford, IL, USA). Equal amounts of protein were separated by $10 \%$ sodium dodecyl sulfate-polyacrylamide gel electrophoresis (SDS-PAGE) and electrophoretically transferred to polyvinylidene fluoride (PVDF) membranes (Millipore, Bedford, MA, USA) using a mini trans-blot (Bio-Rad, Hercules, CA, USA). Membranes were then blocked with PBST (PBS with $0.05 \%$ Tween-20) containing 5\% non-fat dry milk for $1 \mathrm{~h}$ and incubated at $4^{\circ} \mathrm{C}$ overnight with anti-Bcl-2 antibody (Santa Cruz Biotechnology Inc., Santa Cruz, CA, USA), anti-Bax antibody (Cell Signaling Technology, Inc., Beverly, MA, USA) in fresh blocking buffer. Membranes were then washed with PBST and incubated with horseradish peroxidase-conjugated secondary antibody (Santa Cruz Biotechnology) for $1 \mathrm{~h}$. The blots were developed using an enhanced chemiluminescence (ECL) kit (Pierce). Protein levels were normalized against $\beta$-actin (Sigma-Aldrich).

Flow cytometry with rhodamine 123 (Rh123) staining. Mitochondrial membrane potential (MMP) was assessed by the retention of Rh123, a membrane-permeable fluorescent cationic dye. The uptake of Rh123 by mitochondria is proportional to the MMP. Cells were incubated with Rh123 $(0.25 \mathrm{nmol} / \mathrm{l})$ in the dark at room temperature for $20 \mathrm{~min}$. After washing with PBS, the cells were analyzed by FACScan (Becton-Dickinson, San Jose, CA, USA) with excitation and emission wavelengths of 495 and $535 \mathrm{~nm}$, respectively. Cells treated with rapamycin $(10,50$ and $100 \mathrm{nmol} / \mathrm{l})$ for $24 \mathrm{~h}$ were incubated with Rh123 $(0.25 \mathrm{nmol} / \mathrm{l})$ in the dark at room temperature for $20 \mathrm{~min}$. After washing with PBS, the change in MMP was detected by Rh123 staining using flow cytometry.

Electron microscopy. Cells were harvested by scraping them from the plates. They were then washed twice with PBS and fixed with $2 \%$ paraformaldehyde/ $2 \%$ glutaraldehyde in $0.2 \mathrm{M}$ sodium cacodylate buffer ( $\mathrm{pH}$ 7.4). Cell pellets were post-fixed with $1 \%(\mathrm{v} / \mathrm{v})$ osmic acid in sodium cacodylate and stained with $1 \%$ uranyl acetate. Following dehydration, pellets were embedded in Durcopan (Fluka, Sigma). Ultrathin sections were prepared using ULTRACUT S and observed with a JEM 1010 transmission electron microscope. Images were captured and are shown in Results.

Statistical analysis. Data were expressed as mean \pm standard deviation. Mean values were compared using a Student's t-test for independent variables. $\mathrm{P}<0.05$ was considered statistically 

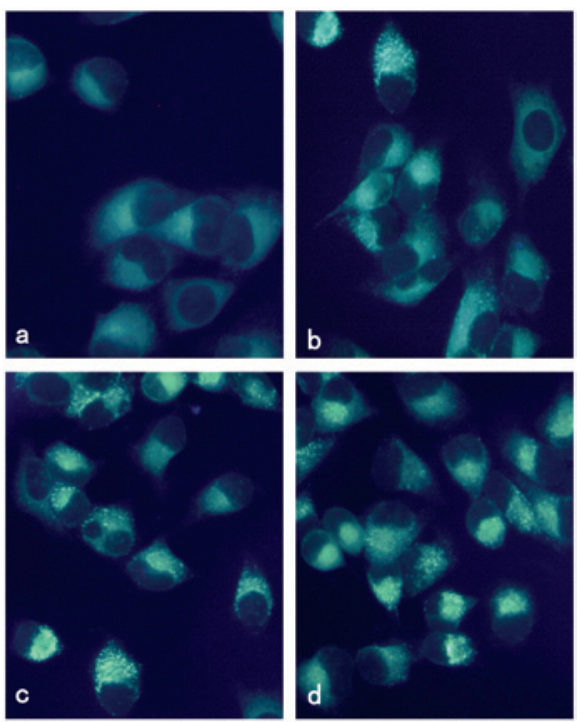

Figure 1. Visualization of autophagy activation with monodansylcadaverine (MDC) in M14 cells. M14 cells were treated with rapamycin (0, 10, 50 or $100 \mathrm{nmol} / \mathrm{l}$ ) and were incubated for $24 \mathrm{~h}$. Cells were stained with MDC as described in Materials and methods. (a) Control group; (b) $10 \mathrm{nmol} / 1$ rapamycin; (c) $50 \mathrm{nmol} / 1$ rapamycin; (d) $100 \mathrm{nmol} / 1$ rapamycin.
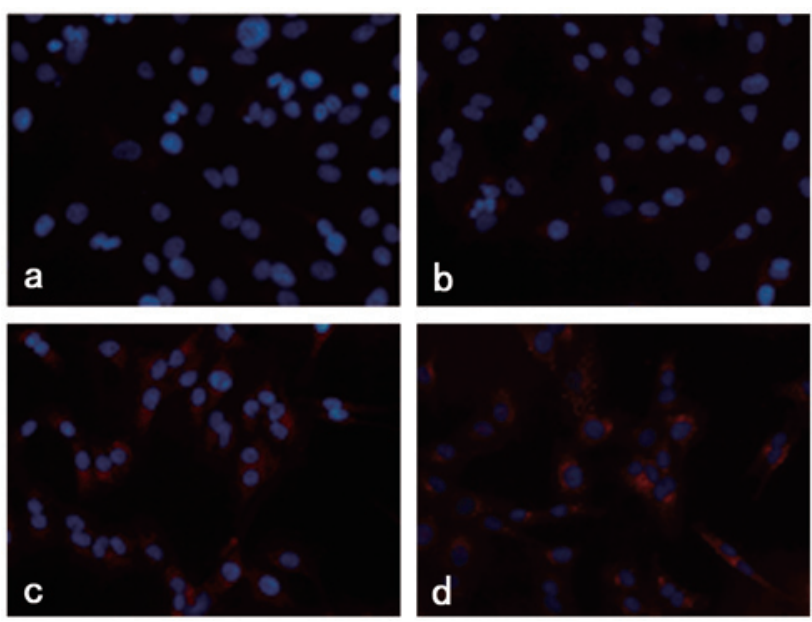

Figure 2. Immunofluorescent staining of LC3B. M14 cells were treated with rapamycin $(0,10,50$ or $100 \mathrm{nmol} / \mathrm{l})$ and were incubated for $24 \mathrm{~h}$. Cells were stained with LC3B as described in Materials and methods. The higher the concentration of rapamycin, the greater the number of fluorescent dots observed. (a) Control group; (b) $10 \mathrm{nmol} / 1$ rapamycin; (c) $50 \mathrm{nmol} / 1 \mathrm{rapamycin}$ and (d) $100 \mathrm{nmol} / 1$ rapamycin.

to indicate a statistically significant difference. All statistical analyses were performed with SPSS 11.0 (SPSS Inc., Chicago, IL, USA).

\section{Results}

Vesicular accumulation of MDC following rapamycin treatment. MDC is an autofluorescent compound and has been proposed as a tracer for autophagic vacuoles. Thus, we studied the incorporation of MDC into M14 cells using fluorescence microscopy. As shown in Fig. 1, M14 cells treated with rapamycin for $24 \mathrm{~h}$ demonstrated a punctate

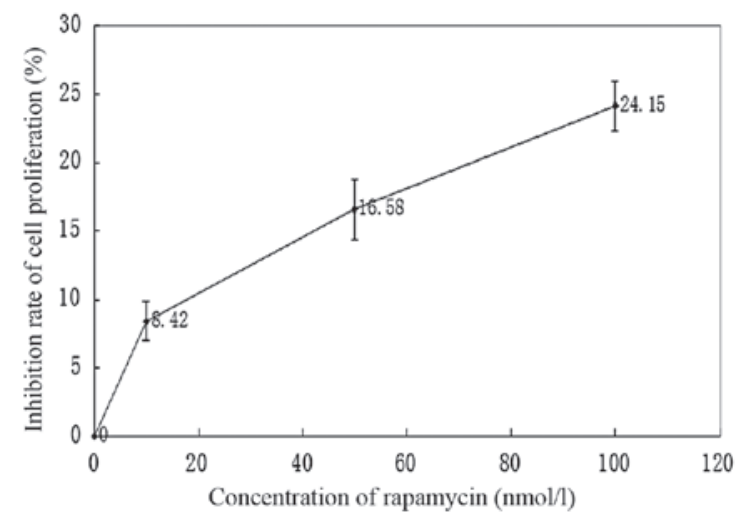

Figure 3. Inhibition rate of proliferation. From $0-100 \mathrm{nmol} / \mathrm{l}$, the inhibition rates of proliferation at rapamycin concentrations of $0,10,50$ and $100 \mathrm{nmol} / 1$ were $0,8.42 \pm 2.88,16.58 \pm 4.43$ and $24.15 \pm 3.69$, respectively. Rapamycin inhibited the proliferation of M14 cells. The inhibition rate of M14 proliferation increased with increasing rapamycin concentration.

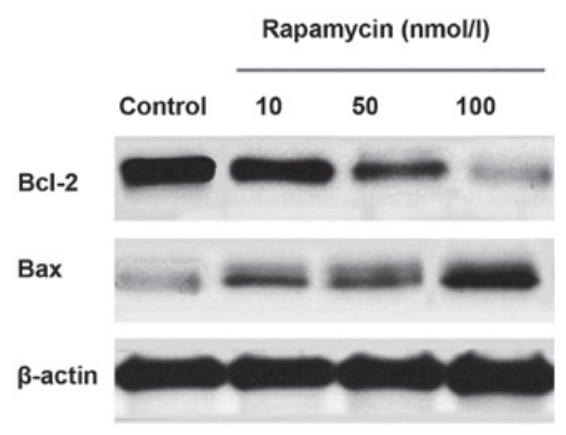

Figure 4. Rapamycin regulated the expression levels of Bcl-2 and Bax. M14 cells were treated with rapamycin $(0,10,50$ or $100 \mathrm{nmol} / \mathrm{l})$ and were incubated for $24 \mathrm{~h}, \mathrm{Bcl}-2$ and Bax expression were assessed by western blot analysis after treatment. Equal amounts of total cellular protein were resolved by $10 \%$ sulfate-polyacrylamide gel electrophoresis (SDS-PAGE) and $\beta$-actin was used as an internal control. The expression of Bcl-2 decreased, while that of Bax increased.

pattern of MDC-labeled fluorescence. By contrast, uninfected cells exhibited a diffused distribution of MDC-labeled fluorescence. Rapamycin induced autophagy of M14 in a concentration-dependent manner.

Rapamycin induced the accumulation of LC3B fluorescence dots in M14 cells. We investigated the effect of rapamycin treatment on the staining of LC3B protein, which is produced during autophagosome formation. As demonstrated in Fig. 2, the quantity of LC3B fluorescence dots increased with increasing rapamycin concentration.

Effects of rapamycin treatment on the proliferation of M14 cells. The proliferation of M14 cells was measured using MTT assay and expressed as a ratio of color intensity from the rapamycin treatment group to that of the DMSO-treated control group. Data were obtained from three independent experimental replicates. Fig. 3 and Table I demonstrated that as the rapamycin concentration increased from $0-100 \mathrm{nmol} / \mathrm{l}$, the strength of the inhibitory effect increased. 
Table I. Effects of rapamycin treatment on the proliferation of M14 cells $(n=4$, mean \pm SD).

\begin{tabular}{lc}
\hline Group & Inhibition rate of proliferation $(\%)$ \\
\hline Control & 0 \\
Rapamycin & \\
$10 \mathrm{nmol} / 1$ & $8.42 \pm 2.88^{\mathrm{a}}$ \\
$50 \mathrm{nmol} / 1$ & $16.58 \pm 4.43^{\mathrm{b}}$ \\
$100 \mathrm{nmol} / 1$ & $24.15 \pm 3.69^{\mathrm{c}}$
\end{tabular}

Rapamycin inhibited the proliferation of M14 cells. M14 cells were treated with rapamycin $(0,10,50$ and $100 \mathrm{nmol} / \mathrm{l})$ and were incubated for $24 \mathrm{~h}$. Cells were stained with monodansylcadaverine (MDC) as detailed in Materials and methods. The P-value between the control and treatment groups was $<0.01$. Each treatment group, the $10 \mathrm{nmol} / 1$ group and the other two groups were significantly different $(\mathrm{P}<0.05)$. However, no significant differences were observed between the 50 and $100 \mathrm{nmol} / \mathrm{l}$ groups $(\mathrm{P}>0.05) .{ }^{\mathrm{a}}$ indicates a statistically significant difference.

Table II. Gray value of Bcl-2 and Bax.

\begin{tabular}{lcc}
\hline Group & Bcl-2 & Bax \\
\hline Control & 1 & 1 \\
Rapamycin & & \\
$10 \mathrm{nmol} / /^{\mathrm{a}}$ & $0.94765 \pm 0.042485$ & $1.96733 \pm 0.097991$ \\
$50 \mathrm{nmol} / \mathrm{l}^{\mathrm{b}}$ & $0.70357 \pm 0.043447$ & $2.23067 \pm 0.372162$ \\
$100 \mathrm{nmol} / \mathrm{l}^{\mathrm{c}}$ & $0.34717 \pm 0.048413$ & $2.89067 \pm 0.208375$
\end{tabular}

The gray value of $\mathrm{Bcl}-2$ and $\mathrm{Bax}$. M14 cells were treated with various concentrations of rapamycin. The gray values of Bcl-2 in 10, 50 and $100 \mathrm{nmol} / \mathrm{l}$ groups were $0.94765 \pm 0.042485,0.70357 \pm 0.043447$ and $0.34717 \pm 0.048413$; those of $B a x$ were $1.96733 \pm 0.097991$, $2.23067 \pm 0.372162$ and $2.89067 \pm 0.208375$. ${ }^{\mathrm{a}} \mathrm{P}>0.05$; ${ }^{\mathrm{b}} \mathrm{P}<0.01$ and ${ }^{\mathrm{C}} \mathrm{P}<0.01$.

Effects of rapamycin treatment on Bcl-2 and Bax expression. M14 cells were treated with various concentrations of rapamycin. Bcl-2 and Bax levels were assayed by western blot analysis. Equal amounts of total cellular protein were resolved by $10 \%$ SDS-PAGE and $\beta$-actin was used as an internal control. As revealed in Fig. 4 and Table II, there was a significant dose-dependent reduction in the level of Bcl-2 expression after rapamycin treatment for $24 \mathrm{~h}$ of 10,50 or $100 \mathrm{nmol} / \mathrm{l}$. By contrast, the level of Bax expression increased.

MMP decreased following rapamycin treatment. Changes in mitochondrial function are capable of launching autophagy. In the case of nutritional deficiencies, there may be mitochondrial permeability transition pores (MPTP), which are a marker of impaired mitochondrial function. Following this, mitochondria are engulfed by lysosomes. A decline in MMP is a morphological characteristic of mitochondrial function recession. $\mathrm{Rh} 123$ is a cationic lipophilic fluorescent dye. It accumulates

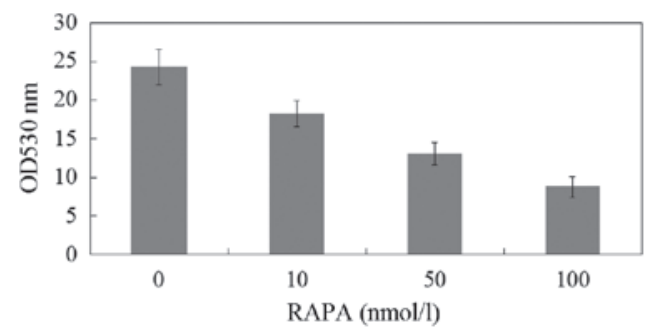

Figure 5. MMP decreased with increasing rapamycin concentration. M14 cells were treated with rapamycin $(0,10,50$ or $100 \mathrm{nmol} / \mathrm{l})$ and were incubated for $24 \mathrm{~h}$. Cells were stained with rhodamine 123 (Rh123) as detailed in Materials and methods. The fluorescence intensity of Rh123 was significantly lower in the cells exposed to rapamycin relative to the control group $(\mathrm{P}<0.05)$. The MMPs of the four groups were $24.26 \pm 2.29,18.24 \pm 1.69,13.11 \pm 1.41$ and $8.79 \pm 1.36$, which indicated that fluorescence intensity decreased with increasing rapamycin concentration. RAPA, rapamycin.

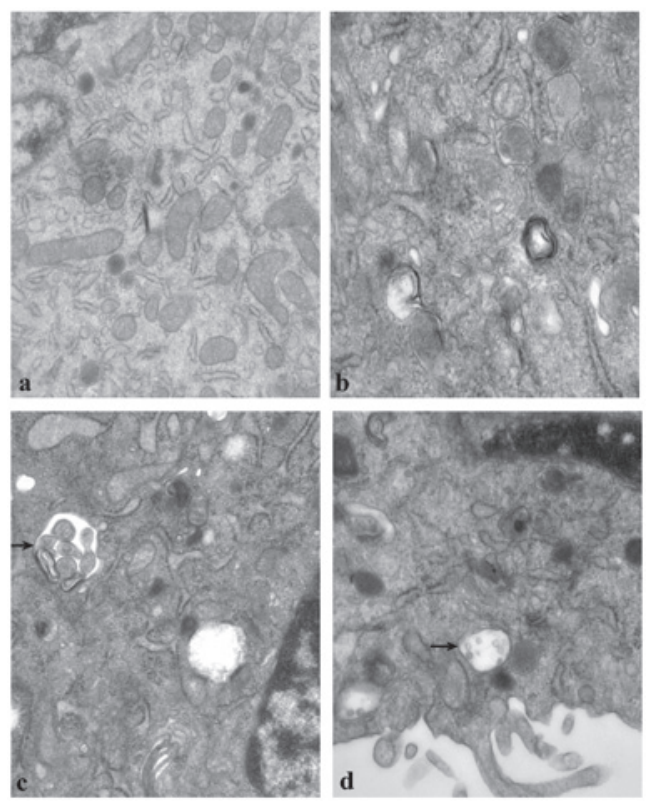

Figure 6. Ultrastructural changes induced by rapamycin. M14 cells were treated with or without rapamycin for $24 \mathrm{~h}$. Ultrastructural changes were observed with a transmission electron microscope. (a) M14 cells were treated without rapamycin and the cellular ultrastructure were normal and (b-d) Cells were treated with $100 \mathrm{nmol} / 1$ rapamycin for $24 \mathrm{~h}$. Autophagosomes (dysfunctional mitochondria sequestered into a double-membrane-bound vesicle) and lysosomes were observed (x40,000).

in mitochondria and its intake is proportional to the MMP. When the MMP decreases, Rh123 in mitochondria leaks out and the fluorescence intensity in the cells reduces. Thus, Rhl23 fluorescence intensity reflects the MMP of mitochondria. As indicated in Fig. 5, the fluorescence intensity of Rh123 was significantly lower in the cells exposed to rapamycin relative to the control group, and the fluorescence intensity decreased with increasing rapamycin concentration.

Ultrastructural examination of autophagy following rapamycin treatment. As demonstrated in Fig. 6, the ultrastructure of the control group (6a) was normal compared with the rapamycin group (6b-d). Double-membrane-bound vesicles, swelling of the mitochondria and vacuolization of 
certain cells were observed in M14 cells following treatment with $100 \mathrm{nmol} / \mathrm{l}$ rapamycin for $24 \mathrm{~h}$. Additionally, abnormal mitochondria, an increased number of lysosomes and autophagosomes were observed.

\section{Discussion}

Melanoma is the most fatal form of skin cancer with increasing incidence throughout the world. There are no efficacious therapies for malignant melanoma at present. It is possible to explore new methods of treatment by modulating autophagy. For example, this may be achieved via rapamycin, an autophagy inducer that promotes autophagy by inhibiting mTOR (9). Interest in applying rapamycin in cancer therapy was initiated due to observations of frequent Akt activation in multiple types of cancer and identification of TSC1/ TSC2 as tumor suppressors that are inhibited by Akt. Cancer therapy with rapamycin has been successfully implemented for kidney cancer, with the approval of CCI-779 (temsirolimus) for the treatment of renal clear cell carcinoma (RCC) (10). In $\mathrm{RCC}$, rapamycin may be effective due to its inhibition of the production of HIF-1 $\alpha$, a key transcription factor that mediates oncogenic alterations in the cellular metabolism $(11,12)$. Aside from RCC, numerous clinical trials are testing rapamycin in cancers linked to Akt activation, such as glioblastoma and prostate cancer. Therefore, in this study, we set out to investigate rapamycin-induced autophagy and its possible mechanisms.

Autophagy is a process by which cells degrade macromolecular intracellular material via sequestration into a double-membranous structure, known as an autophagosome, which then delivers the enclosed material to a lysosome for degradation. Initially believed to be a system dedicated to the 'recycling' of macromolecular material within the cell, autophagy is now known to be involved in a multitude of cellular processes including immunity, tumorigenesis, programmed cell death, the selective degradation of organelles, aging and numerous neurodegenerative conditions (13). There are currently 33 identified autophagy-related genes shown to play a role in autophagy, and many techniques are available to detect autophagy, including transmission electron microscopy, half-life assessments of long-lived proteins, detection of LC3 maturation/aggregation, fluorescence microscopy and co-localization of mitochondrion or endoplasmic reticulum-specific markers with lysosomal proteins (14). In this study, we observed autophagy in M14 cells treated with various concentrations of rapamycin for $24 \mathrm{~h}$ by MDC labeling, LC3B protein staining and transmission electron microscopy. The results showed that rapamycin induced autophagy in M14 cells in a concentration-dependent manner. By using electron microscopy (the most reliable method of observing cell ultrastructure) we observed autophagosomes (dysfunctional mitochondria sequestered into a double-membrane-bound vesicle) and mitochondria that had lost their cristae in cells treated with rapamycin compared with those in the control group. Mitochondria that were abnormal in appearance and and an increased number of lysosomes were also observed.

Mitochondria are vital organelles for cellular metabolism and bioenergetics, but they are also the key regulators of cell death (15). Notably, in many (if not all) paradigms of apoptosis,
MMP represents the point of no return in the cascade of events that ultimately leads to cell death (16). MMP results in the leakage of potentially toxic proteins from the mitochondrial IMS. In addition, mitochondria play a role in stress responses and can produce reactive oxygen species (ROS) when damaged. Selective degradation of mitochondria by autophagy is also known as 'mitophagy' and is considered to be promoted by their functional impairment and/or by MMP. Mitophagy may ensure the removal of damaged and potentially dangerous mitochondria, thus acting as a quality control mechanism. We used Rh123 to detect the changes in MMP by flow cytometry and discovered that the fluorescence intensity of Rh123 was significantly lower in the cells exposed to rapamycin relative to the control group $(\mathrm{P}<0.05)$. Thus, combined with the electron microscopy results, we conclude that rapamycin may affect the regulation mechanism of mitochondria and cause swelling and vacuolization of mitochondria.

The proliferation of M14 cells was measured by the inhibition rate of proliferation using MTT assay (Table II). The results showed that the proliferation of melanoma cells was significantly inhibited in the rapamycin group $(\mathrm{P}<0.01)$. Compared with the other two treatment groups, the $10 \mathrm{nmol} / \mathrm{l}$ group demonstrated notably higher gray values $(\mathrm{P}<0.05)$; however, no significant differences were observed between the $50 \mathrm{nmol} / 1$ and $100 \mathrm{nmol} / \mathrm{l}$ groups $(\mathrm{P}>0.05)$. Thus, it is suggested that rapamycin possesses a biphasic effect. On one hand it is capable of inducing autophagy, on the other hand it may inhibit proliferation.

The correlation between autophagy and apoptosis is complex and controversial (17). It varies with cell type and stress stages. Depending on the cellular context and stimulus, autophagy may be indispensable for apoptosis by initiating the process (18). In other cellular settings, autophagy may rather antagonize or delay apoptosis and inhibition of autophagy may increase the sensitivity of the cells to apoptotic signals (19). In certain cell systems, two processes can occur independently. Numerous signaling pathway overlaps are found between autophagy and apoptosis, including various kinases such as PI3K, PKB/Akt, Bcl-2 family members, PTEN, c-Myc and Ras.

Members of the Bcl-2 family proteins, including Bcl-2, $\mathrm{Bax}$ and $\mathrm{Bak}$, are thought to play regulatory roles in the apoptotic execution of the cells (20). However, more and more studies have revealed that $\mathrm{Bcl}-2$ family proteins also target the autophagy pathway. Further biochemical and genetic data has led to a resurgence of interest in the role of autophagy in tumor suppression (21). In addition to the discovery that an autophagy execution protein, Beclin 1, is a tumor suppressor protein, oncogenic signaling molecules are capable of suppressing autophagy and tumor suppressors are able to stimulate autophagy.

Bcl-2 and Beclin 1 interact in mammalian cells. Firstly, Bcl-2 inhibits Beclin 1-dependent autophagy. To explore the mechanism, an autophagy-competent cell line (HT-29 colon carcinoma cells) that expresses endogenous Beclin 1 has been used. In HT-29 cells, stable transfection of Bcl-2 inhibits starvation-induced autophagy decreases the association of Beclin 1 with Vps34 and decreases the magnitude of Beclin 1-associated class III phosphoinositide-3-kinase activity. This finding suggests that $\mathrm{Bcl}-2$ overexpression blocks the forma- 
tion of the autophagy-promoting Beclin 1-Vps34 complex. Additionally, Bcl-2 inhibits Beclin 1-dependent autophagic cell death. Bcl-2, apoptosis-inhibiting gene and Bax, apoptosis inducing-gene, are two important members of the Bcl-2 family; the ratio between the two determines the survival of cells. There is a negative correlation between the expression of $\mathrm{Bcl}-2$ and Bax. Bcl-2 overexpression leads to cell proliferation, while alternatively Bax leads to cell death. In this study, we investigated the effects of rapamycin treatment on Bcl-2 and Bax expression. As described previously, there was a significant dose-dependent reduction in the level of Bcl-2 expression after rapamycin treatment for $24 \mathrm{~h}$ of 10,50 or $100 \mathrm{nmol} / 1$. By contrast, the level of Bax expression increased. This is speculated to be caused by MPTP. Certain studies suggest that MPTP is formed by the interaction with Bcl-2 family proteins and other proteins outside the mitochondria; Bax may promote the opening of MPTP. Besides, certain authors consider $\mathrm{Bcl}-2$ protein to inhibit the release of $\mathrm{Ca}^{2+}$ from the endoplasmic reticulum, while Bax may promote this release. Rapamycin reduces the expression of $\mathrm{Bcl}-2$ and increases the expression of Bax, which results in an overload of $\mathrm{Ca}^{2+}$ in the mitochondria and promotes the opening of MPTP. Then, mitochondria swell, their outer membranes collapse and exit into the cytoplasm, thus initiating apoptosis.

The ability of Bcl-2 to inhibit autophagy through a direct interaction with Beclin 1 is of particular interest with regards to cancer. The role of autophagy in tumorigenesis and cancer treatment is complex. Autophagy has roles in both tumor prevention and survival, as well as in treatment resistance. As autophagy protects cells from metabolic stress, it is speculated that the upregulation of autophagy preserves cellular fitness and genomic integrity, and thus prevents tumorigenesis (22). On the contrary, established tumor cells utilize autophagy to survive stresses such as nutrient limitation and hypoxia. Furthermore, tumor cells activate autophagy as a stress response to survive cancer treatment. A recent study has demonstrated that under certain situations, such as in radiation-resistant melanoma, inhibition of autophagy may be exploited to prevent resistance to treatment (23). Notably, modulation of autophagy has significant potential in cancer diagnosis and treatment.

At present, there are few studies concerning the effects of rapamycin on melanoma cells; we speculate that rapamycin may be a promising strategy for the effective treatment of melanoma by modulating autophagy and regulating the expression of Bcl-2 family proteins. However, in view of the biphasic effect of rapamycin (in inducing autophagy and apoptosis) there is a requirement to establish the appropriate concentration of rapamycin that is capable of inducing mitophagy, promoting tumor cell apoptosis and activating autophagy, so as to provide a new approach to treating malignant melanoma.

\section{Acknowledgements}

This study was supported by the Science and Technology Social Development Plan of Jiangsu Province (No. BS2007072) and the National Natural Science Foundation of China (No. 81171517).

\section{References}

1. Tang Y, Chen Y, Jiang H and Nie D: Short-chain fatty acids induced autophagy serves as an adaptive strategy for retarding mitochondria-mediated apoptotic cell death. Cell Death Differ 18: 602-618, 2011.

2. Bialik S and Kimchi A: Autophagy and tumor suppression: recent advances in understanding the link between autophagic cell death pathways and tumor development. Adv Exp Med Biol 615: 177-200, 2008.

3. Ouyang D, Zhang Y, Xu L, Li J, Zha Q and He X: Histone deacetylase inhibitor valproic acid sensitizes B16F10 melanoma cells to cucurbitacin B treatment. Acta Biochim Biophys Sin 43: 487-495, 2011

4. Mondesire WH, Jian W, Zhang H, et al: Targeting mammalian target of rapamycin synergistically enhances chemotherapy-induced cytotoxicity in breast cancer cells. Clin Cancer Res 10:7031-7042, 2004.

5. Wu Q, Kiguchi K, Kawamoto T, et al: Therapeutic effect of rapamycin on gallbladder cancer in a transgenic mouse model. Cancer Res 67: 3794-3800, 2007.

6. Yang Z, Lei Z, Li B, et al: Rapamycin inhibits lung metastasis of B16 melanoma cells through down-regulating alphav integrin expression and up-regulating apoptosis signaling. Cancer Sci 101: 494-500, 2010.

7. Youle RJ and Strasser A: The BCL-2 protein family: opposing activities that mediate cell death. Nat Rev Mol Cell Biol 9: 47-59, 2008.

8. Cory S, Huang DC and Adams JM: The Bcl-2 family: roles in cell survival and oncogenesis. Oncogene 22: 8590-8607, 2003.

9. Renna M, Jimenez-Sanchez M, Sarkar S and Rubinsztein DC: Chemical inducers of autophagy that enhance the clearance of mutant proteins in neurodegenerative diseases. J Biol Chem 285: 11061-11067, 2010.

10. Hudes G, Carducci M, Tomczak P, et al: Temsirolimus, interferon alfa, or both for advanced renal-cell carcinoma. N Engl J Med 356: 2271-2281, 2007.

11. Hudson CC, Liu M, Chiang GG, et al: Regulation of hypoxiainducible factor lalpha expression and function by the mammalian target of rapamycin. Mol Cell Biol 22: 7004-7014, 2002.

12. Zhong H, Chiles K, Feldser D, et al: Modulation of hypoxia-inducible factor 1alpha expression by the epidermal growth factor/phosphatidylinositol 3-kinase/PTEN/AKT/FRAP pathway in human prostate cancer cells: implications for tumor angiogenesis and therapeutics. Cancer Res 60: 1541-1545, 2000.

13. Goldman SJ, Taylor R, Zhang Y and Jin S: Autophagy and the degradation of mitochondria. Mitochondrion 10: 309-315, 2010.

14. Tasdemir E, Galluzzi L, Maiuri MC, et al: Methods for assessing autophagy and autophagic cell death. Methods Mol Biol 445: 29-76, 2008.

15. Fantin VR and Leder P: Mitochondriotoxic compounds for cancer therapy. Oncogene 25: 4787-4797, 2006.

16. Kroemer G, Galluzzi L and Brenner C: Mitochondrial membrane permeabilization in cell death. Physiol Rev 87: 99-163, 2007.

17. Laane E, Tamm KP, Buentke E, et al: Cell death induced by dexamethasone in lymphoid leukemia is mediated through initiation of autophagy. Cell Death Differ 16: 1018-1029, 2009.

18. Gozuacik D and Kimchi A: Autophagy as a cell death and tumor suppressor mechanism. Oncogene 23: 2891-2906, 2004.

19. Shi M, Wang HN, Xie ST, et al: Antimicrobial peptaibols, novel suppressors of tumor cells, targeted calcium-mediated apoptosis and autophagy in human hepatocellular carcinoma cells. Mol Cancer 9: 26, 2010.

20. Matsumoto A,Isomoto H, Nakayama M, et al: Helicobacter pylori VacA reduces the cellular expression of STAT3 and pro-survival Bcl-2 family proteins, Bcl-2 and Bcl-XL, leading to apoptosis in gastric epithelial cells. Dig Dis Sci 56: 999-1006, 2011.

21. Pattingre $S$ and Levine B: Bcl-2 inhibition of autophagy: A new route to cancer? Cancer Res 66: 2885-2888, 2006.

22. Eskelinen EL: The dual role of autophagy in cancer. Curr Opin Pharmacol 11: 294-300, 2011.

23. Ravikumar B, Sarkar S, Davies JE, et al: Regulation of mammalian autophagy in physiology and pathophysiology. Physiol Rev 90: 1383-1435, 2010. 